\title{
Acute mitral valve dysfunction and cardiogenic shock due to escape of prosthetic mechanical leaflet
}

\author{
Mirae Lee, Su A Jo, and Ik Hyun Park
}

Department of Internal Medicine, Samsung Changwon Hospital, Sungkyunkwan University School of Medicine, Changwon, Korea

Received: August 26, 2019

Revised : September 19, 2019

Accepted: September 23, 2019

\section{Correspondence to}

Mirae Lee, M.D.

Tel: $+82-55-233-5819$

Fax: +82-55-233-5704

E-mail: conatuse@gmail.com

https://orcid.org/0000-0003-

4142-5403
A 43-year-old man who underwent prosthetic aortic valve and mitral valve (MV) replacement 24 years ago from infective endocarditis was referred to the emergency department for acute dyspnea. On arrival, he was started on mechanical ventilation because of desaturation ( $\mathrm{SpO}_{2}$ 68\%). Tachycardia (178 beats/min), tachypnea (40 breaths/ $\mathrm{min})$, blood pressure of $110 / 90 \mathrm{mmHg}$, and stupor mentality were present. Cardiac murmur was suspected but difficult to confirm. Electrocardiogram showed atrial fibrillation and chest radiography revealed bilateral pulmonary edema. Transthoracic echocardiography showed mildly depressed left ventricular systolic function, no regional wall motion abnormality, and suspicious MV regurgitation. Despite mechanical ventilation and norepinephrine infusion, pulmonary edema and hypotension were aggravated. Emergent extracorporeal membrane oxygenation (ECMO) was performed and allowed for an improvement in the patient's hemodynamic condition. We performed portable transesophageal echocardiography (TEE) to evaluate MV function. TEE showed massive mitral regurgitation extending into the right pulmonic vein. Only a mitral leaflet was observed on two-dimensional images (Fig. $1 \mathrm{~A}, 1 \mathrm{~B}$, and Supplementary Video 1). Three-dimensional images of the MV showed a mobile valve leaflet (Fig. ${ }_{1}$ C, 1 D, and Supplementary Video 2). Emergent MV replacement was performed. Only one mitral leaflet was presented (Fig. 1E) without thrombus or vegetation and the other leaflet was not found. After surgical MV replacement, ECMO and mechanical ventilation could be discontinued on days 2 and 3, respectively. To find the embolized mitral leaflet, we performed a computed tomography scan. Two fragments of the MV leaflet were detected in the left common and external iliac arteries. The fractured mitral leaflet was surgically removed (Fig. $1 \mathrm{~F}$ and $1 \mathrm{G}$ ). The patient recovered uneventfully.

Leaflet escape of the prosthetic valve is an unusual but potentially lethal complication. Although it is rare, when a patient with prosthetic valve presents with acute decompensated heart failure, escape of the valve leaflet should be considered.

Written informed consent by the patient was waived due to a retrospective nature of our report.

\section{Conflict of interest}

No potential conflict of interest relevant to this article was reported. 

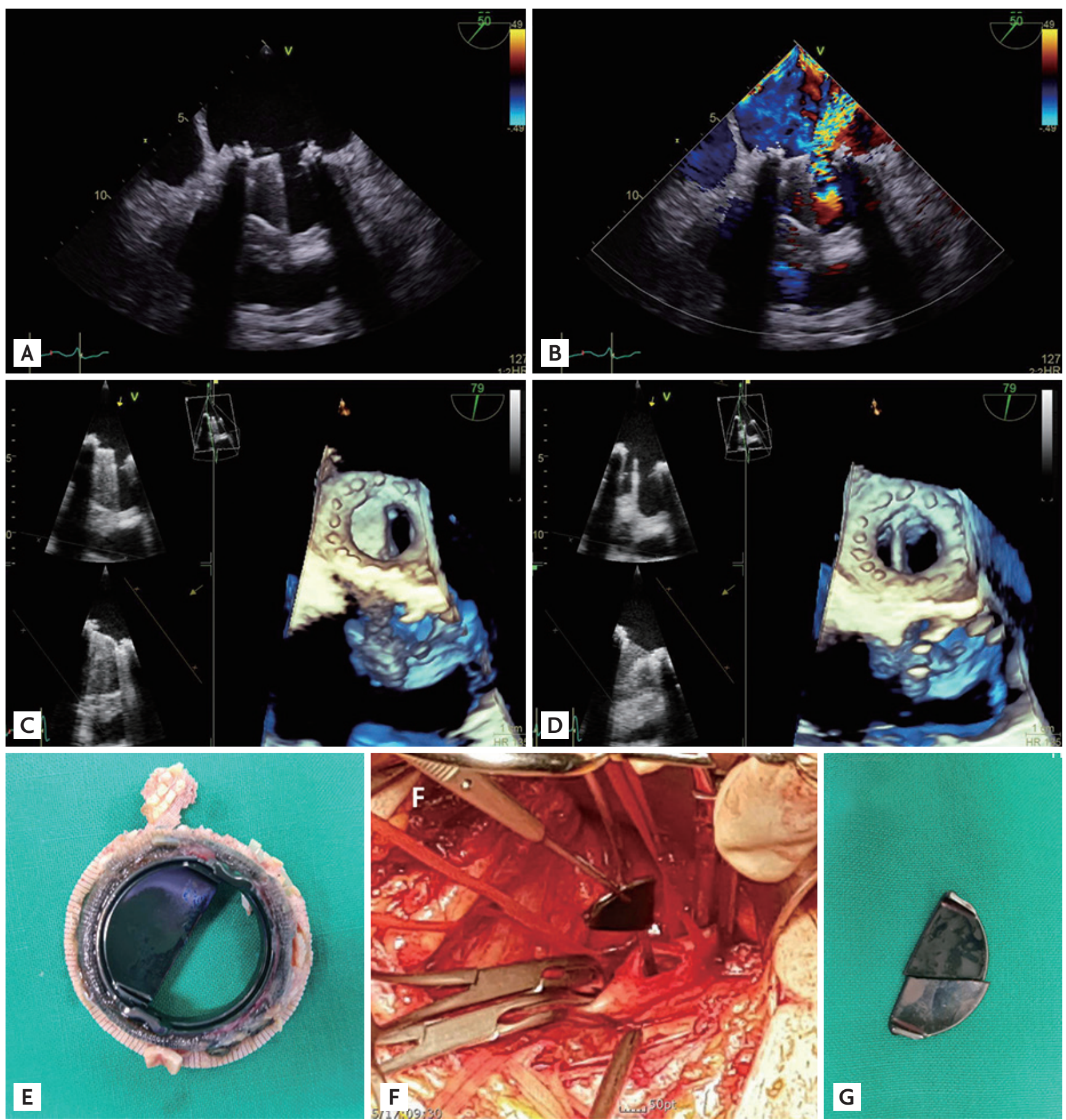

Figure 1. (A-D). Transesophageal echocardiographic images of prosthetic mitral valve. (A) Two-dimensional and (B) color Doppler images. Three-dimensional images in (C) systole and (D) diastole. (E) The surgically removed mitral valve with the single retained leaflet. (F) A fragment of a mitral valve leaflet from the left external iliac artery in the operation field. (G) Two fragments of a mitral valve leaflet removed from the left common and external iliac arteries. 
Supplementary Video 1. The video of the two-dimensional mitral valve image from transesophageal echocardiography. 
Lee $M$, et al. Prosthetic mitral valve leaflet escape

Supplementary Video 2. The video of the three-dimensional mitral valve image from transesophageal echocardiography. 